\title{
Covid 19 Pandemic-Training of Healthcare Workers in Obtaining a Nasopharyngeal Swab: Our Experience
}

\author{
Suma Susan Mathews ${ }^{1}$ (D) ${\text { Lalee } \operatorname{Varghese}^{1} \cdot \text { M. C. Trupthi }}^{1} \cdot$ Nancy Naomi $^{2} \cdot$ \\ Ajoy Mathew Varghese ${ }^{1}$
}

Received: 15 May 2021 / Accepted: 2 September 2021/Published online: 15 September 2021

(C) Association of Otolaryngologists of India 2021

\begin{abstract}
To describe the training of healthcare workers (HCW) in nasopharyngeal swabbing during the COVID 19 pandemic. Study design: Retrospective study. Setting: Tertiary care teaching hospital. Subjects and methods: One hundred and seventy eight health care workers were trained from May 2020 to January 2021. Three modules were designed to train the health care workers in the technique of obtaining a nasopharyngeal swab specimen.Training consisted of an instructional video on how to perform nasopharyngeal swabs and live demonstration followed by hands-on supervised training. The trainees included 30 doctors, 101 nurses, 31 respiratory therapists, 3 physiotherapists, 9 interns and 4 lab technicians. There were 39 male and 139 female trainees. After attending all 3 modules of training, they were confident and efficient in taking a non-traumatic nasopharyngeal swab. Good knowledge and adequate training is key to a good nasopharyngeal sampling for SARS CoV-2 testing.
\end{abstract}

Keywords Nasopharyngeal swab - SARS CoV-2 . COVID 19 pandemic . Health care worker

Suma Susan Mathews

sumasusanm@yahoo.co.in

1 Department of ENT, Christian Medical College, Vellore, Tamil Nadu 632004, India

2 College of Nursing, Christian Medical College, Vellore, Tamil Nadu, India

\section{Introduction}

COVID-19, an infectious disease caused by the severe acute respiratory syndrome coronavirus 2 (SARS-CoV-2), is mainly transmitted through droplets generated when an infected person coughs, sneezes, or exhales. WHO recommended sampling the respiratory material for detection of the virus in suspected cases [1]. Different testing methods used in the detection of active SARS-CoV-2 infection include molecular tests (real-time reverse transcription polymerase chain reaction or RT-PCR) and antigen tests. RT-PCR remains the mainstay because of its high sensitivity and specificity [2-4]. With a high viral load in the upper respiratory tract during the first week of symptoms, the yield of nasopharyngeal swabs is superior to oropharyngeal swabs [5]. Tagliabu et al. highlighted the role of the otolaryngologist in training and guidance of personnel for nasopharyngeal sampling [6].

Being a large tertiary care university-affiliated teaching hospital, our institution was identified as a COVID care centre for our district. The protocols and guidelines for managing COVID patients were drawn by the institutional guideline development team. The ENT doctors were entrusted with the task of obtaining the nasopharyngeal swabs for testing. With an allotment of about 800 beds for management of the COVID patients as well as outpatient screening and testing of contacts and suspects, there was a felt need to train other health care workers on the technique of nasopharyngeal sample collection. We report our experience with the training of health care professionals in nasopharyngeal swabbing. 


\section{Objective and Methodology}

\section{Objective}

To describe the training of healthcare workers (HCW) in nasopharyngeal swabbing.

\section{Methodology}

Three modules were designed to train the health care workers in the technique of obtaining a nasopharyngeal swab specimen (Table 1). Training consisted of an instructional video on how to perform nasopharyngeal swabs and live demonstration followed by hands-on supervised training.

\section{MODULE I: (Location-CLASSROOM)}

\section{Part 1-Power point presentation:}

a. General Instructions on the Nasopharyngeal Swab Technique The session began with a brief description of the anatomy of the nasal cavity and nasopharynx with figures/pictures. A video recording of the technique of obtaining a nasopharyngeal swab, using a flocked nylon tipped swab with a flexible shaft, on a mannequin was played as instructions were given (Fig. 1). The trainees were instructed on the correct way of introducing the swab along the floor of the nasal cavity and parallel to the palate after stabilizing the head and advancing it to about twothirds of the distance between the nose and ear lobule, till a resistance to further advancement was encountered. If there was resistance before that and was causing discomfort to the patient, they were advised to withdraw the swab and maneuver it once again to the correct length or obtain a sample from the other nasal cavity. The importance of not introducing the swab vertically was emphasized (to avoid encountering the turbinates or breaching the skull base).The likelihood of obtaining an inaccurate sample if the swab was introduced only for a few centimetres into the nose was emphasized. Instruction was given to leave the

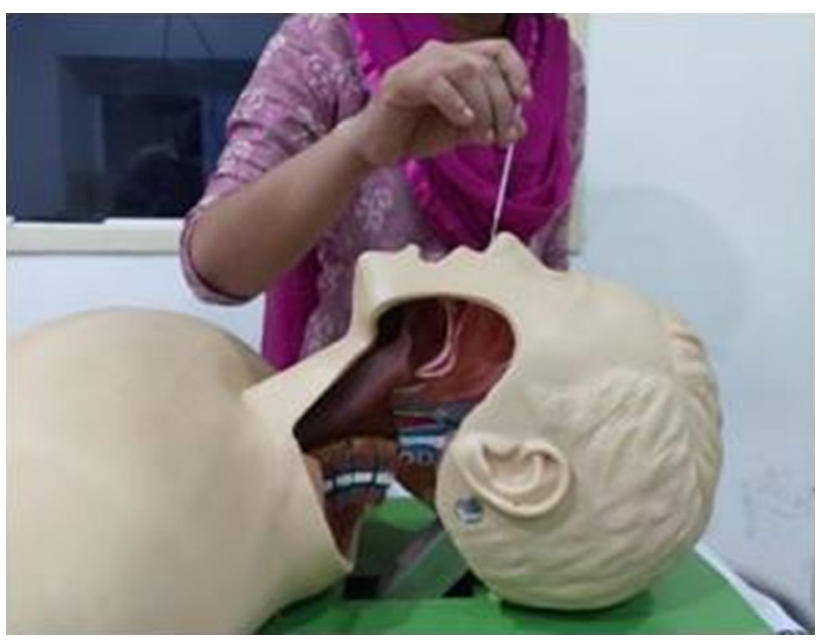

Fig. 1 Demonstration on mannequin

swab in place for 5 to $10 \mathrm{~s}$ (to saturate the tip) once it was in place, rotate the swab once and then remove it slowly.

\section{Management of complications}

The participants were informed of possible complications and their management. In the event of any post-procedure nasal bleeds, the trainees were instructed to ask the patient to sit and lean forwards and pinch the nose with the thumb and index finger for $5 \mathrm{~min}$. If epistaxis persisted, the on-call duty ENT doctor was to be paged.

b. Instructions for Obtaining a Swab in the Ward Donning: Wearing appropriate personal protective equipment including disposable gown, head cape, overshoes, protective N95 mask, face shield and nonsterile gloves.

Pre-procedure check: informed consent, appropriate Indian Council of Medical Research Specimen Referral Form and brief check if the patient had any bleeding disorder or deranged bleeding parameters.

Nasopharyngeal swabbing: A video recording of the procedure being performed on a patient in the ward was then played. The patient wears a surgical mask with a small hole for the nostril, through which the swab would be introduced into the nasal cavity (Fig. 2). The mask reduces the spread of aerosol generated during the procedure.

Table 1 Nasopharyngeal sampling training programme

\begin{tabular}{|c|c|c|c|c|c|}
\hline Module & Venue & Duration & Trainees per session & Resource person & Content \\
\hline Module 1 & Classroom & $30 \mathrm{~min}$ & $10-15$ & ENT & $\begin{array}{l}\text { Lecture with videos- } 15 \text { min } \\
\text { Hands on training-Mannequin and POP model of the head }\end{array}$ \\
\hline Module 2 & Inpatient ward & $2 \mathrm{~h}$ & $3-4$ & ENT & $\begin{array}{l}\text { Hands on training-supervised } \\
\text { Observe } 1 \text {, perform } 3\end{array}$ \\
\hline Module 3 & Swabbing Kiosk & $15-30 \mathrm{~min}$ & $2-3$ & ENT & $\begin{array}{l}\text { Hands on training-supervised } \\
\text { Observe } 1 \text {, perform } 2\end{array}$ \\
\hline
\end{tabular}


Handling the specimen: The chaperone/assistant opens the labelled tube with the transport medium and swab placed in the tube after which the handle of the flocked nylon tipped swab is broken off and the tube capped.

c. Instructions for Obtaining a Swab From the Kiosk The trainees were then informed about the kiosk for outpatient swabbing of suspects and contacts. A customized swabbing kiosk was constructed for the purpose (Fig. 3). The kiosk is a $2 \times 1.5 \times 1.5 \mathrm{~m}$ booth with a protective glass panel in the front. The panel had built in glove holder to fix the gloves which the HCW dons prior to taking the swab. It also included a two-way mike for communication between the HCW and the patient. The health care operator donning the PPE would enter the kiosk, introduce his gloved hands and forearm into the fixed gloves while the patient to be swabbed would stand on the other side of the glass pane. The transport medium containing tube and the swab is handed over to the $\mathrm{HCW}$, who then proceeds to obtain the sample. After each procedure, the rubber gloves, outer walls of the kiosk and the surrounding area were disinfected by the orderly in PPE. A video recording of the procedure was played.

\section{Part 2 Hands on Training on the Nose Model and the Mannequin}

The HCW first saw the procedure being done on the mannequin with a sagittal section view showing the nasal cavity and the nasopharynx (Fig. 1). As they practiced a couple of times on the Plaster of Paris nose model (Fig. 4)

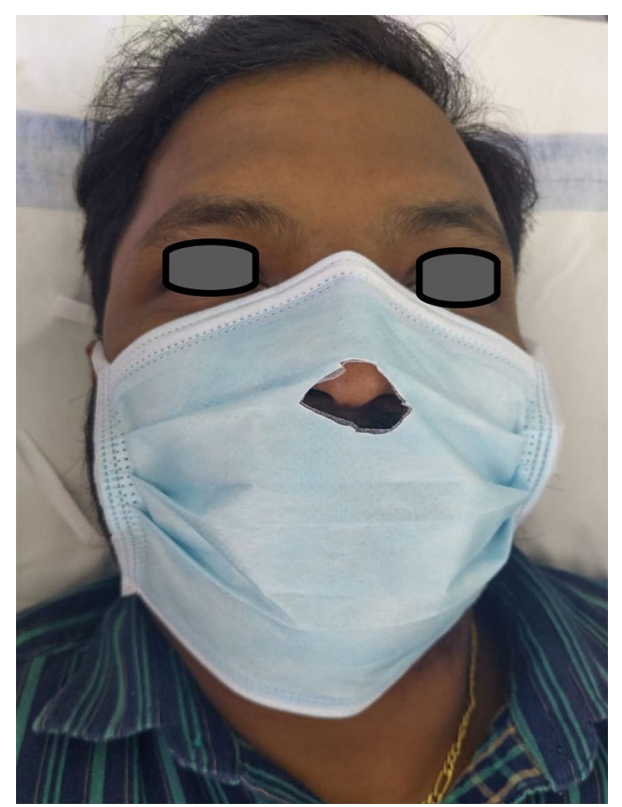

Fig. 2 Bedside training they were familiarized with the nuances of overcoming obstructions in the nasal cavity and passing the swab to reach the nasopharyngeal space. Further confidence was gained when the trainee performed the procedure on a mannequin at the end of the session.

\section{MODULE 2: (Location-INPATIENT WARD)}

The second module training was in the wards where swab was done for in-patients. A trainer (ENT doctor), demonstrated the technique of obtaining a sample on a patient admitted for the swabbing to 3 or $4 \mathrm{HCW}$ at a time. Each trainee then performed the procedure under supervision on at least 3 patients and the trainer certified the HCW to independently perform the swabbing.

\section{MODULE 3: (Location-OUTPATIENT KIOSK)}

In this module, the trainees would observe the trainer (ENT doctor) swabbing a patient and then perform it under supervision on 2 patients. The trainer then certified the $\mathrm{HCW}$ to independently perform the procedure.

\section{Results}

A total of 178 health care workers were trained from May 2020 to January 2021. The trainees included 30 doctors, 101 nurses, 31 respiratory therapists, 3 physiotherapists, 9 interns and 4 lab technicians. There were 39 male and 139 female trainees. After attending all 3 modules of training, they were confident and efficient in taking a non-traumatic nasopharyngeal swab.

\section{Discussion}

On 11 March 2020, WHO declared the Novel Corona virus Disease (COVID-19) outbreak as a global pandemic, and as the number of people with active infection increased, to fight the pandemic, the 'Test, Track and Treat' strategy was adopted [7]. Methods developed to detect COVID-19 infection rapidly and efficiently included Molecular (RTPCR) tests, COVID-19 Antigen Tests and COVID-19 serology testing. RT-PCR test is regarded as the gold standard of testing while rapid antigen testing (RAT) is a faster but less reliable method. In our institution the RTPCR testing is being done on a regular basis and RAT introduced for testing only in an emergency.

The clinical specimens for RT-PCR can be obtained from upper respiratory tract (nasopharyngeal swabs, washes, aspirates, or oropharyngeal swabs) or from lower respiratory tract (sputum collection, bronchoalveolar 
Fig. 3 Swabbing kiosk

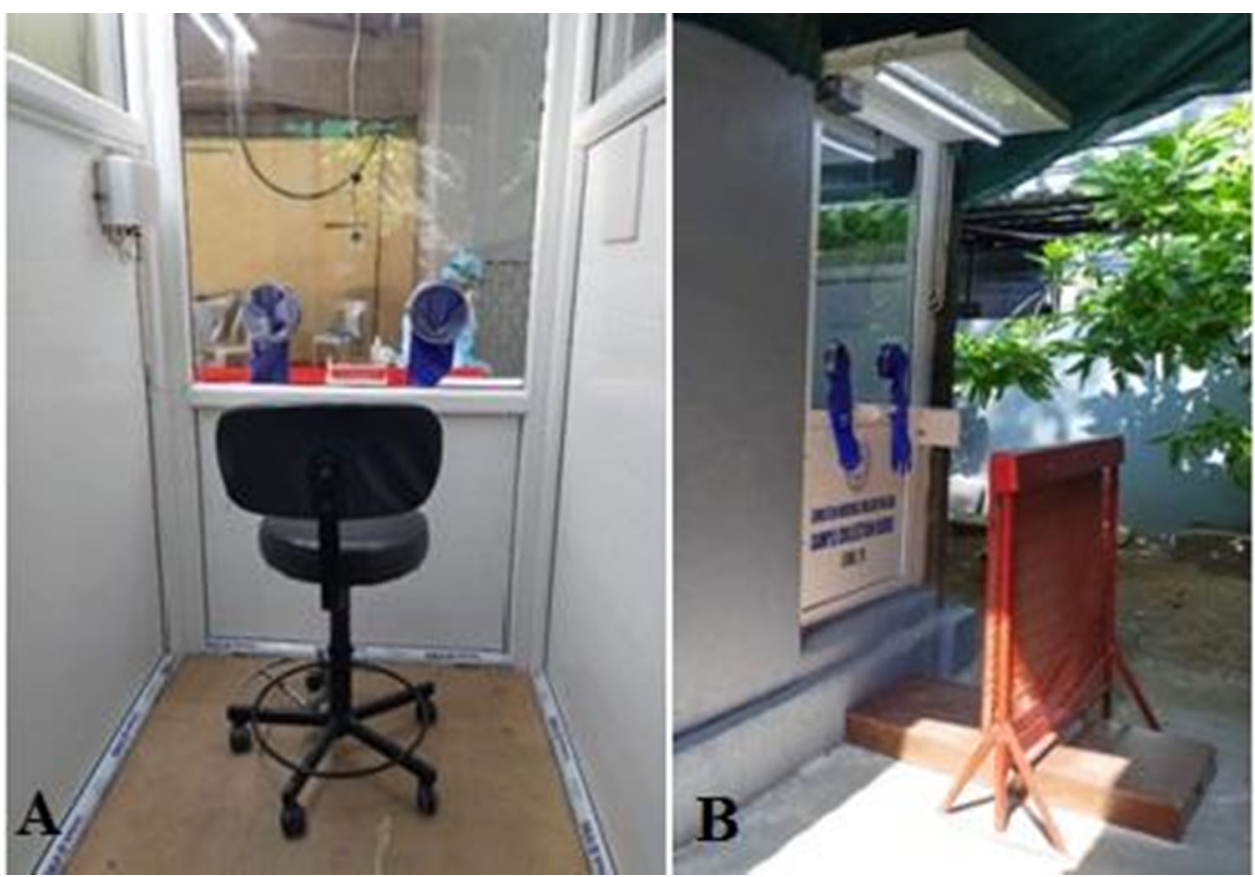

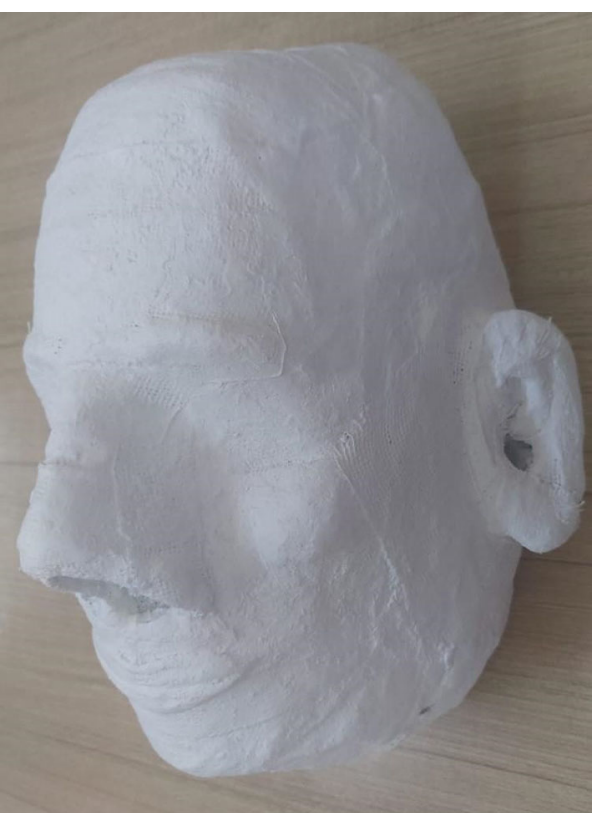

Fig. 4 Plaster of Paris nose model for hands on training

lavage (BAL) or tracheal aspirates) [8]. Of these, nasopharynx showed a much higher viral load and swabs from nasopharynx were more sensitive. However inappropriate nasopharyngeal sampling has been shown to be a reason for false negative reports [9].

Good anatomic knowledge and procedural skill are vital for an adequate high quality nasopharyngeal specimen. Otolaryngologists are more oriented to anatomy of nose and nasopharynx and were the primary clinicians involved in nasopharyngeal sampling. The very high number of patients requiring nasopharyngeal sampling and need to make it available at multiple points required more personnel. Hence, the department of otolaryngology took the initiative to train other health care workers by making a training programme with 3 modules and were able to effectively train the HCW for a standardized sampling technique.

Video demonstrations have been used in other centers for training the HCW [10]. The strength of our training programme is that in addition to video and demonstration, the comprehensive training included hands-on supervised training before a $\mathrm{HCW}$ was certified to be capable of independently performing the procedure. This made them more confident in the sampling process. At the end of module 1 , all the trainees were familiar with the anatomy of nose and nasopharynx, technique of nasopharyngeal swabbing in the ward as well as in the kiosk and also had a hands-on training on the mannequin. In module 2 and 3 they had supervised onsite experience before being certified to independently do the nasopharyngeal sampling.

\section{Conclusion}

Good knowledge and adequate training is key to a good nasopharyngeal sampling for SARS CoV-2 testing. On completion of all three modules, the HCW became more confident as they performed the procedure under 
supervision. Acquiring a new skill, the HCW felt empowered and their confidence was boosted as they felt they could also contribute and play a part in the management of the pandemic.

Funding This work was partially supported by the institution.

\section{Declarations}

Conflict of interest The authors have no conflict of interest to disclose in this work.

\section{References}

1. World Health Organization (2020) Laboratory testing for 2019 novel coronavirus (2019-nCoV) in suspected human cases. Interim guidance. WHO/COVID-19/laboratory/2020.5. World Health Organization, Geneva, Switzerland. https://www.who.int/ publications-detail/laboratory-testing-for-2019-novel-coronavirus -in-suspected-human-cases-20200117.

2. McFee DRB (2020) COVID-19 laboratory testing/CDC guidelines. Dis Mon. 66(9):101067

3. Lu H, Stratton CW, Tang YW (2020) An evolving approach to the laboratory assessment of COVID-19. J Med Virol 92:1812-7
4. Islam KU, Iqbal J (2020) An update on molecular diagnostics for COVID-19. Front Cell Infect Microbiol 10:560616

5. Mawaddah A, Gendeh HS, Lum SG, Marina MB (2020) Upper respiratory tract sampling in COVID-19. Malays J Pathol 42:23-35

6. Tagliabue M, Pietrobon G, Ugolini S, Chu F, Ansarin M (2020) Nasopharyngeal swabs during SARS-CoV-2 pandemic: a role for the otolaryngologist. Eur Arch Otorhinolaryngol 277:2155-2157

7. Novel Coronavirus (2019-nCoV) situation report - 1. World Health Organization (2020) Jan 21, [2020-04-05]. https://www. who.int/docs/default-source/coronaviruse/situation-reports/20200 121-sitrep-1-2019-ncov.pdf.

8. Hung KF, Sun YC, Chen BH, Lo JF, Cheng CM, Chen CY et al (2020) New COVID-19 saliva based test: how good is it compared with the current nasopharyngeal or throat swab test? J Chin Med Assoc 83:981-894

9. Piras A, Rizzo D, Uzzau S, De Riu G, Rubino S, Bussu F (2020) Inappropriate nasopharyngeal sampling for SARS-CoV-2 detection is a relevant cause of false-negative reports. Otolaryngol Head Neck Surg 163:459-61

10. Coronavirus disease: What you need to know. World Health Organization. https://www.afro.who.int/news/coronavirus-dise ase-what-you-need-know.

Publisher's Note Springer Nature remains neutral with regard to jurisdictional claims in published maps and institutional affiliations. 